\title{
Editorial
}

\section{Need for Managing Variability of Healthcare Processes}

Hospitals have scarce capacity. Almost always the demand for care is higher than the supply provided. Managing a hospital organization in an efficient, patientcentered, and timely way is easier said than done.

Let us try to understand the fundamentals of this problem. Imagine an 'ideal' world where a typical hospital provides services to patients. This hospital has 800 beds, 25 operating rooms (ORs), 50 intensive care unit (ICU) beds. Everyday exactly 80 patients are admitted and the length of stay (LOS) is exactly 10 days. All patients admitted are equal and all patients undergo the same procedure in the ORs. This procedure will be performed every time by the same surgical team. The procedure time is exactly 155 minutes and, after admission, every patient spends exactly 2

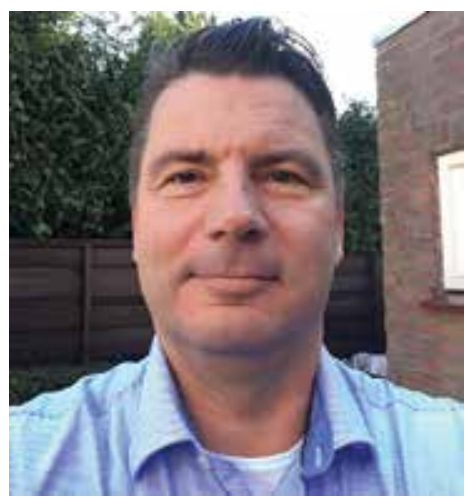
hours in the ICU and is discharged after exactly 10 days. In this world, hospital management can offer a perfect, efficient, patient-centered and timely service to the patient. Number of beds in uses will be, in the long-term, exactly 800 beds. There is no overtime in the ORs because all the procedures have the same duration, and the professionals in the ICU should not only know exactly when they can expect the next patient but also when this very same patient will be transported to the ward.

This ideal world is utopia. In healthcare, the word 'exactly' does not exist. To compare a hospital with a wine factory, there is known exactly how many bottles are filled with wine every minute and how many bottles are produced at the end of a working day. And at $5 \mathrm{pm}$, machines stop working. In healthcare, working days stop technically at $5 \mathrm{pm}$. But at $5 \mathrm{pm}$, we do not close the ORs when still patients are being operated.

In our example, remove the word 'exactly', and replace it by 'mean'. Now, in our real daily business, we call it a hospital where all patients are coming for the same or different procedures and surgical teams differ everyday and every week. From the fundamental point of view, we introduced the concept of 'variability'. Variability in health processes means that patients have to wait in queues for their appointment in the outpatient department, surgical procedures take more time than expected, patients stay shorter or longer in the ICU and the LOS may be shorter or longer. The latter results sometimes in that new patients cannot be admitted because of overloaded wards, where professionals work under stress. And, this may subsequently cause idle time in the ORs resulting in revenue loss for the hospital.

Managing variability can be a nightmare for many hospital managers. But, it should not be this way. Of course, everybody will understand that it is not possible to eliminate all the variability and, hence, create our ideal world (which is still utopia). But, we can use common sense and apply scientifically proven tools to at least diminish variability in healthcare processes and, hence, approach more toward a situation where efficient, patient-centered and timely care are more or less in an equilibrium.

Why should we do this at all, managing variability? Firstly, we want to use our scarce and costly hospital resources in an optimal way by knowing what is in the system and what can be expected. And secondly, we want to make sure that the service to our patient is timely by reducing the chance that a patient is rejected for admission or that a scheduled surgery is canceled. It is amazing to see that air traffic control knows from every plane where it is and when it is expected to take off and land. Ask yourself: do you know at this very moment (or within 5 minutes) how many elective patients are admitted to your hospital today and will be admitted for next Tuesday? And, how many patients you might expect tomorrow at 8 pm in the emergency room? Amazing, no!

Healthcare operation management is a medicine to handle to a certain extent the variability within healthcare processes. Basically, healthcare operations management focuses on the delivery of safe care to our patients. This is performed by analyzing the underlying operations and processes, thereby taking into account the potential links with resources (wards, ICUs, ORs, labs) needed.

Next we describe some examples. Concerning the OR, start the first case on time. A delay on the first case means that there is a higher probability that the last case will be cancelled because of overtime. Check your OR schedule per room. Try to schedule cases in one OR such that the sum of the variance for that OR is minimal. This gives a higher probability that all scheduled cases can be finished. 
A simple example to illustrate this point. Assume block time OR number one is 10 hours (600 minutes). We have the option to schedule a combination of the following surgeries (mean procedure time and standard deviation (SD) calculated per surgeon-procedure combination): knee replacement (120 minutes, SD 30 minutes), pancreatectomy (300 minutes, SD 70 minutes), laparoscopic cholecystectomy (60 minutes, SD 15). Assume further that the mean turn over time between cases is 15 minutes.

For OR number 15 we can create two possible schedules:

Schedule A: Two patients for knee replacement followed by a pancreatectomy. Total time needed: procedure time 540 minutes plus 30 minutes turn over time equal 570 minutes. The sum of variances (square of standard deviation) of the included procedures equals 6,700.

Schedule B: Three patients for a knee replacement, followed by three patients for a laparoscopic cholecystectomy. Total time needed 615 minutes. Total variance: 3,3750.

Expected variance schedule B is smaller than schedule A, hence we prefer schedule A.

Another interesting but sometimes hard to answer question is, how many beds needed in the ward? A very useful tool can be found here http://www.vumc.nl/afdelingen/pica/Software/erlang_b/ Here we use the Erlang B loss mode. The Erlang B is also used in call centers to calculate how many operators are needed to let the caller not to wait more than let us say 3 minutes (with a probability of 95\%). Input variables are: Arrivals, Average Stay, Operational/Staffed beds in the ward. Output data are: rejection rate, occupancy rate. Arrivals and average length of stay can be calculated using historical data of a specific ward. Suppose the number of arrivals per day is 5 patients, staying on average 4.5 days and that the ward has 30 beds. Then the percentage of refused admissions is $2.47 \%$ while the occupancy rate is $73.15 \%$. More beds reduces the percentage of refused admissions but decreases the occupancy rate. Reducing beds increases the occupancy rate but may also create increased workload for the staff.

In these examples health operations management can help. Because of the mathematics and statistics involved it is sometimes hard to follow. But trying to understand this and applying it subsequently in your hospital brings your hospital more in control and hence will answer the previous questions in an affirmative way.

Pieter Stepaniak PhD Professor Catharina Hospital Eindhoven, The Netherlands Medical University of Gdańsk, Poland 